\title{
The Lorentz group and its finite field analogues: local isomorphism and approximation
}

\author{
Stephan Foldes \\ Tampere University of Technology \\ PL 553, 33101 Tampere, Finland \\ sf@tut.fi \\ 4 August 2008
}

\begin{abstract}
Finite Lorentz groups acting on 4-dimensional vector spaces coordinatized by finite fields with a prime number of elements are represented as homomorphic images of countable, rational subgroups of the Lorentz group acting on real 4-dimensional space-time. Bounded subsets of the real Lorentz group are retractable with arbitrary precision to finite subsets of such rational subgroups. These finite retracts correspond, via local isomorphisms, to well-behaved subsets of Lorentz groups over finite fields. This establishes a relationship of approximation between the real Lorentz group and Lorentz groups over very large finite fields.
\end{abstract}

\section{Finite Minkowski spaces and statement of the theorem}

The purpose of this paper is to establish a relationship of approximation between Lorentz transformations of Minkowski space $\mathbb{R}^{4}$ and Lorentz transformations of 4-dimensional spaces over very large finite fields, in the precise sense of Theorem 1 below. In relation to theoretical alternatives in particle physics, Lorentz transformations over finite fields seem to have been first considered by Coish in [C], then by Shapiro [S], Ahmavaara [A1, A2], Yahia [Y], Joos [Jo], Beltrametti and Blasi [BB1], and Nambu [N]. Much of the algebra of Lorentz groups over finite fields had already been developed by Dickson [D], and in the context of physical applications this theory was brought up to date by Beltrametti and Blasi [BB2]. A finite analogue of the Alexandrov-Zeeman

characterization of the Lorentz group via optical causality was established 
by Blasi, Gallone, Zecca and Gorini [BGZG]. The approximation described in Theorem 1 relies on the one hand on modifying a relationship of approximation between positive real numbers and quadratic residues modulo a large prime established by Kustaanheimo [JK, K1, K2], and modifying Järnefelt's approximation of collinear ranges of points in Euclidean space by collinear ranges in finite geometries [Jä]. The proof of Theorem 1 also makes essential use of rotation-boost decomposition, and of representing Lorentz groups over finite fields as quotients of appropriate subgroups of the real Lorentz group. The algebraic background and terminology used in this theorem are as follows.

We consider fields $\mathbf{F}$ in which -1 is not a square, and we shall assume throughout that $\mathbf{F}$ has this property. (The main cases of interest are the field $\mathbb{R}$ of real numbers and the $p$-element finite fields $\mathbf{F}_{p}$ for prime numbers $p \equiv 7 \bmod 8$, although many of the basic facts are also true for $p$-element fields with primes $p \equiv 3 \bmod 8$.) For any such field $\mathbf{F}$ the 4-dimensional vector space $\mathbf{F}^{4}$ is endowed with the (F-valued) Minkowski norm

$$
\mu(t, x, y, z)=t^{2}-x^{2}-y^{2}-z^{2}
$$

A proper Lorentz transformation over $\mathbf{F}$ is a linear transformation $T$ from the vector space $\mathbf{F}^{4}$ to itself which preserves the Minkowski norm $($ i.e., $\mu(T(\mathbf{v}))=$ $\mu(\mathbf{v})$ for all $\mathbf{v} \in \mathbf{F}^{4}$ ) and which has determinant 1. For any given field $\mathbf{F}$, these transformations form a group that we call the proper Lorentz group over $\mathbf{F}$, denoted $\mathcal{L}_{+} \mathbf{F}$. If the field $\mathbf{F}$ is not specified, it is understood to be $\mathbb{R}$. A Lorentz transformation over a field $\mathbf{F}$ is said to be orthochronous if it maps $(1,0,0,0)$ to a vector $(t, x, y, z)$ where $t$ is a non-zero square in $\mathbf{F}$. For $\mathbf{F}=\mathbb{R}$ orthochronous Lorentz transformations form a subgroup of $\mathcal{L}_{+} \mathbb{R}$ called the orthochronous proper Lorentz group, denoted $\mathcal{L}_{+}^{\uparrow} \mathbb{R}$. Over finite fields the composition of orthochronous transformations is not necessarily orthochronous.

If $G$ and $H$ are two (abstract) groups and $A \subseteq G, Y \subseteq H$ are arbitrary sets of group elements, a local isomorphism between $A$ and $Y$ is a bijective map $\sigma$ from $A \cup A A$ to $Y \cup Y Y$ (where $A A=\{x y: x, y \in A\}$ ) such that $\sigma[A]=Y$ and for all $x, y \in A$

$$
\sigma(x y)=\sigma(x) \sigma(y)
$$


It follows that if $1_{G} \in A$ then $\sigma\left(1_{G}\right)=1_{H}$ and if $x, x^{-1} \in A$ then $\sigma\left(x^{-1}\right)=$ $\sigma(x)^{-1}$. If such a local isomorphism exists, the sets $A$ and $Y$ are said to be locally isomorphic. (If $A$ and $Y$ are subgroups they are locally isomorphic if and only if they are isomorphic, but otherwise the sets $A$ and $Y$ may be locally isomorphic even if the subgroups they generate are not isomorphic.)

By the norm $\|T\|$ of any linear transformation $T: \mathbb{R}^{4} \longrightarrow \mathbb{R}^{4}$ with standard matrix representation $\left(a_{i j}\right)_{1 \leq i, j \leq 4}$ we mean $\left(\Sigma a_{i, j}^{2}\right)^{1 / 2}$, i.e. the Euclidean $\left(l_{2}\right)$ norm of the matrix. By a retraction of $\mathcal{L}_{+}^{\uparrow} \mathbb{R}$ to a subset $A \subseteq \mathcal{L}_{+}^{\uparrow} \mathbb{R}$ we mean a map $f: \mathcal{L}_{+}^{\uparrow} \mathbb{R} \longrightarrow A$ such that $f(T)=T$ for all $T \in A$ (i.e., such that $\left.f^{2}=f\right)$.

Theorem 1 For every $\epsilon>0$ and $M>0$ the orthochronous proper Lorentz group $\mathcal{L}_{+}^{\uparrow} \mathbb{R}$ has a retraction $f: \mathcal{L}_{+}^{\uparrow} \mathbb{R} \longrightarrow A$ to a finite subset $A \subseteq \mathcal{L}_{+}^{\uparrow} \mathbb{R}$ satisfying

$$
\|T-f(T)\|<\epsilon
$$

for all Lorentz transformations $T \in \mathcal{L}_{+}^{\uparrow} \mathbb{R}$ of norm not exceeding $M$, and such that for some prime number $p \equiv 7 \bmod 8$ the subset $A$ is locally isomorphic to a set $Y$ of orthochronous transformations in the proper Lorentz group $\mathcal{L}_{+} \mathbf{F}_{p}$ over the finite field $\mathbf{F}_{p}$.

The proof is provided in Section 2, together with some elaborations of the mathematical aspects that are needed for the sake of precision and clarity. These are presented as a succession of intermediate propositions. A generalization to the extended Lorentz group is presented in Theorem 2 of Section 3, with some additional properties of the local isomorphism linking the respective Lorentz groups over the real numbers and over finite fields. In Section 4 we briefly comment on some of the particularities of finite analogues of the Lorentz group.

\section{Background developments and proof of Theorem 1}

\subsection{Proper Lorentz groups over fields where -1 is not a square}

Much of the basic theory of finite Lorentz groups that we need is contained in or follows from the seminal work of Dickson $[\mathrm{D}]$ and the more recent study of Beltrametti and Blasi [BB2]. 
Lorentz groups are defined according to the active perspective as groups of bijective transformations, and composition of transformations is denoted simply by juxtaposition $T_{1} T_{2}$, where $T_{2}$ is the transformation first applied. If the standard matrix representations of these transformations are $\mathbf{T}_{1}$ and $\mathbf{T}_{2}$ then the matrix product $\mathbf{T}_{1} \mathbf{T}_{2}$ represents the transformation $T_{1} T_{2}$.

A proper Lorentz transformation $T$ over a field $\mathbf{F}$ where -1 is not a square is called a space rotation (over $\mathbf{F}$ ) if it fixes the first standard basis vector $(1,0,0,0) \in \mathbf{F}^{4}$. Equivalently, space rotations over $\mathbf{F}$ are the linear transformations of $\mathbf{F}^{4}$ with standard matrix representation of the block diagonal form

$$
\left(\begin{array}{ll}
1 & \\
& \mathbf{R}_{3}
\end{array}\right)
$$

where $\mathbf{R}_{3}$ is a 3 -by-3 orthogonal matrix (i.e. $\mathbf{R}_{3}^{\top}=\mathbf{R}_{3}^{-1}$ ) with determinant 1. There are three space rotations which permute the standard basis vectors, they are given by

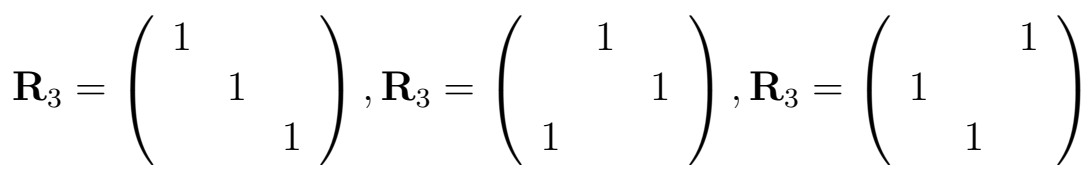

These particular space rotations are called rotations of standard space axes and they form a 3-element subgroup of $\mathcal{L}_{+} \mathbf{F}$. All space rotations constitute a subgroup of $\mathcal{L}_{+} \mathbf{F}$ over any field $\mathbf{F}$. If a space rotation fixes one of the three standard space unit vectors $(0,1,0,0),(0,0,1,0)$ or $(0,0,0,1)$ then it is said to be an elementary space rotation (around that unit vector). For each of these three unit vectors, elementary space rotations around that vector constitute a subgroup of the group of all space rotations. Around $(0,1,0,0)$ this subgroup consists of the identity, the half-turn around $(0,1,0,0)$ given by

$$
(t, x, y, z) \mapsto(t, x,-y-z)
$$

and for each $\alpha \neq 0$ in $\mathbf{F}$ the transformation $R_{\alpha}$ called a basic rotation whose standard matrix representation is

$$
\left(\begin{array}{llll}
1 & & & \\
& 1 & & \\
& & \frac{\alpha-\alpha^{-1}}{\alpha+\alpha^{-1}} & \frac{2}{\alpha+\alpha^{-1}} \\
& & \frac{-2}{\alpha+\alpha^{-1}} & \frac{\alpha-\alpha^{-1}}{\alpha+\alpha^{-1}}
\end{array}\right)
$$


Each of the two groups of elementary space rotations around $(0,0,1,0)$ and $(0,0,0,1)$ is obtained from the group of elementary space rotations around $(0,1,0,0)$ by conjugation with space axis rotations represented by appropriate permutation matrices. All space rotations are orthochronous, over any field $\mathbf{F}$.

For every non-zero square $\alpha$ in $\mathbf{F}$ (which means positive $\alpha$ in $\mathbb{R}$, quadratic residue class in $\mathbb{Z} / p \mathbb{Z} \cong \mathbf{F}_{p}$ ) the basic boost $B_{\alpha}$ is defined as the Lorentz transformation over $\mathbf{F}$ with standard matrix representation

$$
\left(\begin{array}{cccc}
\frac{\alpha+\alpha^{-1}}{2} & \frac{\alpha-\alpha^{-1}}{2} & & \\
\frac{\alpha-\alpha^{-1}}{2} & \frac{\alpha+\alpha^{-1}}{2} & & \\
& & 1 & \\
& & & 1
\end{array}\right)
$$

For any space rotation $R$ and $\alpha$ any non-zero square in $\mathbf{F}$, the conjugate $R B_{\alpha} R^{-1}$ is called a boost, and for any given space rotation $R$ the set of boosts

$$
\left\{R B_{\alpha} R^{-1}: \alpha \neq 0, \alpha \text { square in } \mathbf{F}\right\}
$$

is a subgroup of $\mathcal{L}_{+} \mathbf{F}$. If the space rotation $R$ is a rotation of standard space axes, then $R B_{\alpha} R^{-1}$ is said to be an elementary boost. A boost is elementary if and only if it fixes two of the three basis vectors $(0,1,0,0),(0,0,1,0),(0,0,0,1)$. All boosts are orthochronous over $\mathbb{R}$, but boosts over a finite field do not need to be orthochronous: consider over $\mathbf{F}_{7}$ any of the two non-trivial boosts $B_{2}$ and $B_{4}$.

Over any field $\mathbf{F}$, note that composing space-time reversal

$$
(t, x, y, z) \mapsto(-t,-x,-y,-z)
$$

with the half-turn around $(0,1,0,0)$ yields reflection in the yz plane given by

$$
(t, x, y, z) \mapsto(-t,-x, y, z)
$$

Basic boosts together with this reflection generate the subgroup of $\mathcal{L}_{+} \mathbf{F}$ fixing $(0,0,1,0)$ and $(0,0,0,1)$. (This subgroup is also referred to as the group of hyperbolic isometries of the $t x$ plane.)

Since every space rotation in $\mathcal{L}_{+} \mathbb{R}$ is the product of 3 elementary space rotations, elementary space rotations and basic boosts generate $\mathcal{L}_{+}^{\uparrow} \mathbb{R}$, and 
together with reflection in the $y z$ plane they generate $\mathcal{L}_{+} \mathbb{R}$. On the other hand, a classical result on linear groups over finite fields due to Dickson [D] states, when particularized to the case of 4-dimensional spaces over $\mathbf{F}_{p}$ for primes $p \equiv 7 \bmod 8$, that $\mathcal{L}_{+} \mathbf{F}_{p}$ is generated by elementary rotations, elementary boosts and reflections in the $y z$ plane. Re-stated in a slightly strengthened form, based on the observation that all elementary boosts are conjugates of basic boosts by axis rotations which are in turn products of elementary space rotations, we have the following:

Rotation-Boost Lemma over Finite Fields (from Dickson [D]) For any prime number $p \equiv 7 \bmod 8$, the proper Lorentz group $\mathcal{L}_{+} \mathbf{F}_{p}$ is generated by basic boosts, elementary space rotations and space-time reversal.

In fact the above lemma also holds for primes $p \equiv 3 \bmod 8$ with the exception of $p=3$ (see [D]) but this fact is not needed in what follows.

Unlike over the real number field $\mathbb{R}$, over finite fields not every proper Lorentz transformation can be represented in the form $R B$ (or $B R$ ) with a rotation $R$ and a boost $B$. (Any such product of a rotation and a boost transforms $(1,0,0,0)$ to a vector $(t, x, y, z)$ where $x^{2}+y^{2}+z^{2}$ is a square in the field of scalars. However, over finite fields not all proper Lorentz transformations, not even the orthochronous ones, have this property.) For the real case see Moretti's study $[\mathrm{M}]$ for a comprehensive perspective on rotation-boost decomposition.

\subsection{Lorentz transformations over local rings}

We represent the $p$-element field $\mathbf{F}_{p}$ as the quotient of the localization ring

$$
\mathbb{Z}_{(p)}=\mathbb{Z} \cdot(\mathbb{Z} \backslash p \mathbb{Z})=\left\{s t^{-1}: s, t \in \mathbb{Z}, \quad t \neq 0,(p, t)=1\right\}
$$

by its unique maximal ideal $p \mathbb{Z}_{(p)}$, an approach also taken by Ahmavaara [A1]. There is a unique surjective ring homomorphism from the localization ring $\mathbb{Z}_{(p)}$ onto the $p$-element field $\mathbf{F}_{p}$, called the canonical map (or canonical surjection) from $\mathbb{Z}_{(p)}$ to $\mathbf{F}_{p}$. (The restriction of the canonical map to $\mathbb{Z} \subseteq$ $\mathbb{Z}_{(p)}$ is the unique surjective ring homomorphism $\mathbb{Z} \longrightarrow \mathbf{F}_{p}$ corresponding to the usual representation of $\mathbf{F}_{p}$ as a quotient of $\mathbb{Z}$.) The canonical map from $\mathbb{Z}_{(p)}$ to $\mathbf{F}_{p}$ induces a surjective ring homomorphism from the ring of 4-by-4 matrices with entries in $\mathbb{Z}_{(p)}$ onto the ring of 4 -by-4 matrices with entries in 
$\mathbf{F}_{p}$ and thus induces a map from any set of linear transformations of $\mathbb{R}^{4}$ with coefficients in $\mathbb{Z}_{(p)}$ to the set of linear transformations of $\mathbf{F}_{p}^{4}$

For any integral domain $\mathbf{D}$ in which -1 is not a square, those $\mathbf{D}$-module automorphisms $L$ of $\mathbf{D}^{4}$ whose standard $4 \times 4$ matrix representation has determinant 1 and which leave invariant the (D-valued) Minkowski norm

$$
\mu(t, x, y, z)=t^{2}-x^{2}-y^{2}-z^{2}
$$

are called Lorentz transformations over $\mathbf{D}$ and they constitute a group, called the proper Lorentz group over $\mathbf{D}$, denoted $\mathcal{L}_{+} \mathbf{D}$. A transformation $L$ in $\mathcal{L}_{+} \mathbf{D}$ is said to be orthochronous if it maps $(1,0,0,0)$ to a vector $(t, x, y, z)$ such that $t$ is a non-zero square in $\mathbf{D}$. The set $\mathcal{L}_{+}^{\uparrow} \mathbf{D}$ of such orthochronous transformations may or may not be a subgroup of $\mathcal{L}_{+} \mathbf{D}$. A D-module automorphism $L$ of $\mathbf{D}^{4}$ having determinant 1 belongs to $\mathcal{L}_{+} \mathbf{D}$ if and only if its standard matrix representation $\mathbf{L}$ and the diagonal matrix $\mathbf{J}$ with main diagonal $(1,-1,-1,-1)$ satisfy the equation $\mathbf{L} \mathbf{J L}^{\top}=\mathbf{J}$. For any set $C$ of elements of the integral domain $\mathbf{D}$, we denote by $\mathcal{L}_{+} \mathbf{D} C$, respectively by $\mathcal{L}_{+}^{\uparrow} \mathbf{D} C$, the set of those $L \in \mathcal{L}_{+} \mathbf{D}$, respectively $L \in \mathcal{L}_{+}^{\uparrow} \mathbf{D}$, for which all entries of the standard matrix representation of $L$ are in $C$. If $\mathbf{C}$ is a subring of $\mathbf{D}$ (containing the unit 1 of $\mathbf{D}$ ) then $\mathcal{L}_{+} \mathbf{D C}$ is a subgroup of $\mathcal{L}_{+} \mathbf{D}$. and restricting transformations $L \in \mathcal{L}_{+} \mathbf{D C}$ to $\mathbf{C}^{4} \subseteq \mathbf{D}^{4}$ yields a group isomorphism, called canonical isomorphism,

$$
\rho: \mathcal{L}_{+} \mathrm{DC} \longrightarrow \mathcal{L}_{+} \mathbf{C}
$$

(The closure of $\mathcal{L}_{+} \mathbf{D C}$ under inversion is due to the fact that for all $L \in \mathcal{L}_{+} \mathbf{D}$ with matrix $\mathbf{L}$ we have $\mathbf{L}^{-1}=\mathbf{J L}^{\top} \mathbf{J}$.) The isomorphism $\rho: \mathcal{L}_{+} \mathbf{D C} \longrightarrow \mathcal{L}_{+} \mathbf{C}$ obviously maps orthochronous transformations to orthochronous transformations, $\rho\left[\mathcal{L}_{+}^{\uparrow} \mathbf{D C}\right]=\mathcal{L}_{+}^{\uparrow} \mathbf{C}$, and $\mathcal{L}_{+}^{\uparrow} \mathbf{D C}$ and $\mathcal{L}_{+}^{\uparrow} \mathbf{C}$ are locally isomorphic subsets even if they are not subgroups of $\mathcal{L}_{+} \mathbf{D C}$ and $\mathcal{L}_{+} \mathbf{C}$. They are isomorphic subgroups, however, in the case of particular interest, which is $\mathbf{D}=\mathbb{R}$ and $\mathbf{C}=\mathbb{Z}_{(p)}$ (for any $\left.p \equiv 3 \bmod 4\right)$. The following lemma provides a representation of the proper Lorentz group over a finite field $\mathbf{F}_{p}$ as a quotient of the proper Lorentz group over the localization ring $\mathbb{Z}_{(p)}$, or equivalently, as a quotient of the group of (real) proper Lorentz transformations with coefficients in $\mathbb{Z}_{(p)}$.

Homomorphism Lemma Let $p$ be any prime number congruent to 7 modulo 8. The canonical map $\mathbb{Z}_{(p)} \longrightarrow \mathbf{F}_{p}$ induces a surjective group homo- 
morphism $\mathcal{L}_{+} \mathbb{Z}_{(p)} \longrightarrow \mathcal{L}_{+} \mathbf{F}_{p}$. Pre-composing it with the canonical isomorphism $\rho$ yields a surjective group homomorphism $\mathcal{L}_{+} \mathbb{R} \mathbb{Z}_{(p)} \longrightarrow \mathcal{L}_{+} \mathbf{F}_{p}$.

Proof Its is obvious that the map $\mathcal{L}_{+} \mathbb{Z}_{(p)} \longrightarrow \mathcal{L}_{+} \mathbf{F}_{p}$ induced by the canonical map $\mathbb{Z}_{(p)} \longrightarrow \mathbf{F}_{p}$ is a group homomorphism. In $\mathcal{L}_{+} \mathbf{F}_{p}$ every basic boost, every elementary rotation and also the space-time reversal transformation is in the range of this homomorphism. Its surjectivity is then a consequence of the Rotation-Boost Lemma over Finite Fields.

The two homomorphisms onto $\mathcal{L}_{+} \mathbf{F}_{p}$ defined by the above lemma will be also referred to as canonical.

\subsection{Local isomorphisms}

The next few lemmas are easily verified.

Injection Lemma 1 If $h: G_{1} \longrightarrow G_{2}$ is a group homomorphism, $A \subseteq$ $G_{1}$ and the restriction $h_{A}$ of $h$ to $A \cup A A$ is injective, then $h_{A}$ is a local isomorphism between $A$ and $h[A]$.

Ahmavaara [A1] seems to have been aware of the fact stated in Injection Lemma 1, at least in the particular case where $h$ is the canonical surjection $\mathbb{Z}_{(p)} \longrightarrow \mathbf{F}_{p}$ viewed as an additive group homomorphism, while apparently disregarding (or treating differently) the case where $h$ is the restriction of the same canonical map to $\mathbb{Z}_{(p)}^{*}=\mathbb{Z}_{(p)} \backslash p \mathbb{Z}_{(p)}$ and viewed as a multiplicative group homomorphism onto $\mathbf{F}_{p}^{*}=\mathbf{F}_{p} \backslash\{0\}$.

For each positive integer $k$ consider the set $C_{k}$ of rational numbers defined by

$$
C_{k}=\left\{s t^{-1}: s, t \in \mathbb{Z}, \quad t \neq 0,|s| \leq k,|t| \leq k\right\}
$$

If $p$ is a prime number larger than $k$, then $C_{k} \subseteq \mathbb{Z}_{(p)}$.

Injection Lemma 2 For any prime number $p \equiv 3 \bmod 4$ let $k$ be a positive integer such that $2 k^{2}<p$. Then the canonical map $\mathbb{Z}_{(p)} \longrightarrow \mathbf{F}_{p}$ is injective on $C_{k}$ and the canonical map $\mathcal{L}_{+} \mathbb{R} \mathbb{Z}_{(p)} \longrightarrow \mathcal{L}_{+} \mathbf{F}_{p}$ is injective on $\mathcal{L}_{+} \mathbb{R} C_{k}$.

Injection Lemma 3 For any prime number $p \equiv 3 \bmod 4$ let $k$ be a positive integer such that $32 k^{16}<p$. Let $A=\mathcal{L}_{+} \mathbb{R} C_{k}$. Then the canonical homomorphism $\mathcal{L}_{+} \mathbb{R}_{(p)} \longrightarrow \mathcal{L}_{+} \mathbf{F}_{p}$ is injective on $A \cup A A$. 
Proof $A \cup A A \subseteq \mathcal{L}_{+} \mathbb{R} C_{4 k^{8}}$ and Injection Lemma 2 .

These yield the following

Local Isomorphism Lemma For every positive integer $k$ and prime number $p \equiv 3 \bmod 4$ such that $p>32 k^{16}$, the set $\mathcal{L}_{+} \mathbb{R} C_{k}$ of proper Lorentz transformations with coefficients in $C_{k}$ is locally isomorphic to a subset of the finite proper Lorentz group $\mathcal{L}_{+} \mathbf{F}_{p}$. A local isomorphism is provided by restricting the canonical homomorphism $\mathcal{L}_{+} \mathbb{R} \mathbb{Z}_{(p)} \longrightarrow \mathcal{L}_{+} \mathbf{F}_{p}$ to $\mathcal{L}_{+} \mathbb{R} C_{k}$.

Using Dirichlet's theorem on primes in arithmetic progressions, in [K1] Kustaanheimo proved the following:

Kustaanheimo's Chain Theorem [K1] For every positive integer $k$ there is a prime number $p>k, p \equiv 7 \bmod 8$, such that all the non-negative integers up to $k$ are quadratic residues modulo $p$. There are infinitely many such primes $p$ for any given $k$, they are necessarily larger than $2 k$.

From this we derive a key fact about the sets $C_{k}$, noting that these are obtained from $\{1,2, \ldots, k\}$ by symmetrization via the adjunction of negatives and reciprocals:

Symmetrized Chain Lemma For every positive integer $k$ there is a prime number $p>k, p \equiv 7 \bmod 8$, such that all the positive numbers in $C_{k} \subseteq \mathbb{Z}_{(p)}$ are quotients $a / b$ of quadratic residues $a, b$ modulo $p$, i.e. all positive members of $C_{k}$ are mapped to non-zero squares in $\mathbf{F}_{p}$ by the canonical surjection $\mathbb{Z}_{(p)} \longrightarrow \mathbf{F}_{p}$.

Proof By the Kustaanheimo Chain Theorem there is a prime number $p>k^{2}, p \equiv 7 \bmod 8$, such that all the non-negative integers up to $k^{2}$ are quadratic residues modulo $p$.

\subsection{Density and conclusion of proof of the theorem}

By the coefficients of a linear transformation of $\mathbb{R}^{n}$ we mean the entries of its standard matrix representation.

In this Section we deal mainly with the metric space structure on the set of all linear transformations of $\mathbb{R}^{4}$ induced by the Euclidean norm of standard matrix representations as briefly introduced in Section 1. In this 
metric space, the distance between two transformations $T, Q$ is the norm of their difference, $\|T-Q\|$.

In $\mathcal{L}_{+}^{\uparrow}=\mathcal{L}_{+}^{\uparrow} \mathbb{R}$ the norm of any space rotation is 2 and the norm of the basic boost $B_{\alpha}$ is $\alpha+\alpha^{-1}$. If $T$ is any linear transformation of $\mathbb{R}^{4}$ and $R$ is any space rotation, then $\|T\|=\|R T\|=\|T R\|$. The norm of a proper orthochronous Lorentz transformation $T=R S B_{\alpha} S$ in $\mathcal{L}_{+}^{\uparrow}$, where $R, S$ are space rotations, is $\alpha+\alpha^{-1} \geq 2$, and this is also the norm of the inverse transformation $T^{-1}$.

In any metric space, a set $A$ of elements is said to be $\epsilon$-dense in a set of elements $B$ (where $\epsilon$ is any positive real) if for every $b \in B$ there is an $a \in A \cap B$ at distance less than $\epsilon$ from $b$. Note that this does not require $A$ to be a subset of $B$, but $A$ is $\epsilon$-dense in $B$ if and only if $A \cap B$ is $\epsilon$-dense in $B$. Note that if $A$ is $\epsilon$-dense in a set $B$, and also in a set $C$, then $A$ is $\epsilon$-dense in $B \cup C$, but the converse implication generally fails. Density in the usual sense means $\epsilon$-density for all $\epsilon>0$.

Approximation Lemma 1 For every positive integer $k$ the set $C_{k^{2}}$ is $(1 / k)$-dense in the real interval $[-k, k]$.

Proof This follows from the inclusion

$$
\left\{i / k: i \in \mathbb{Z} \quad-k^{2} \leq i \leq k^{2}\right\} \subseteq C_{k^{2}} \cap[-k, k]
$$

Approximation Lemma 2 Let $\epsilon>0$ and let $K$ be the union of a finite number of non-trivial compact real intervals. Then there is a positive integer $l$ such that for all integers $k \geq l$ the set $C_{k}$ is $\epsilon$-dense in $K$.

Proof Approximation Lemma 1.

Approximation Lemma 3 Let $\epsilon>0$ and let $\mathbf{u}$ be any of the three standard space unit vectors. Then there is a positive integer $l$ such that for all integers $k \geq l$ the set $\mathcal{L}_{+} \mathbb{R} C_{k}$ is $\epsilon$-dense within the set of space rotations around $\mathbf{u}$.

Proof Suppose $\mathbf{u}=(0,1,0,0)$. In the other cases the proof follows by conjugation.

The identity transformation and the half-turn around $\mathbf{u}$ have their coefficients in $C_{k}$.

There is some $M>0$ such that for all basic rotations $R_{\alpha}$ with $M<\alpha$ the distance between $R_{\alpha}$ and the identity is less than $\epsilon$. 
There is some $0<\mu<M$ such that for all basic rotations $R_{\alpha}$ with $0<\alpha<\mu$ the distance between $R_{\alpha}$ the and the half-turn is less than $\epsilon$.

Let $K=[-M,-\mu] \cup[\mu, M]$. By uniform continuity of the map $\alpha \mapsto R_{\alpha}$ on the compact set $K$, there is $\delta>0$ such that for all $\alpha, \gamma \in K,|\alpha-\gamma|<\delta$ implies $\left\|R_{\alpha}-R_{\gamma}\right\|<\epsilon$.

By Approximation Lemma 2, there is a positive integer $l$ such that for all integers $k \geq l$ the set $C_{k}$ is $\delta$-dense in $K$.

Let $k \geq l$ and let $R_{\alpha}$ be a basic space rotation with $\alpha \in K$. By $\delta$-density there is a $\gamma \in C_{k} \cap K$ such that $|\alpha-\gamma|<\delta$. We have $\left\|R_{\alpha}-R_{\gamma}\right\|<\epsilon$.

Approximation Lemma 4 Let $\epsilon>0$ and $M>0$. There is a positive integer $l$ such that for all integers $k \geq l$ the set $\mathcal{L}_{+} \mathbb{R} C_{k}$ is $\epsilon$-dense within the set of basic boosts of norm not exceeding $M$.

Proof There is some $0<\mu<M$ such that all basic boosts $B_{\alpha}$ with $\alpha<\mu$ have norm greater than $M$.

Let $K=[\mu \cup M]$. By uniform continuity of the map $\alpha \mapsto B_{\alpha}$ on the compact interval $K$, there is $\delta>0$ such that for all $\alpha, \gamma \in K,|\alpha-\gamma|<\delta$ implies $\left\|B_{\alpha}-B_{\gamma}\right\|<\epsilon$.

By Approximation Lemma 2, there is a positive integer $l$ such that for all integers $k \geq l$ the set $C_{k}$ is $\delta$-dense in $K$.

Let $k \geq l$ and let $B_{\alpha}$ be a basic boost with $\alpha \in K$. By $\delta$-density there is a $\gamma \in C_{k} \cap K$ such that $|\alpha-\gamma|<\delta$. We have $\left\|B_{\alpha}-B_{\gamma}\right\|<\epsilon$.

The above lemmas combine to yield the following

Approximation Lemma 5 Let $\epsilon>0$ and $M>0$. There is a positive integer $l$ such that for all integers $k \geq l$ the set $\mathcal{L}_{+} \mathbb{R} C_{k}$ is $\epsilon$-dense within each of the three elementary space rotation groups around the standard space unit vectors, and also within the set of basic boosts of norm not exceeding $M$.

Approximation Lemma 6 Let $\epsilon>0$ and $M>0$. There is a positive integer $l$ such that for all integers $k \geq l$ the set $\mathcal{L}_{+} \mathbb{R} C_{k}$ is $\epsilon$-dense within the set of orthochronous proper Lorentz transformations of norm not exceeding $M$. 
Proof By a compactness and uniform continuity argument, there is a $\delta>0$ such that for all linear transformations

$$
T_{1}, \ldots, T_{10}, Q_{1}, \ldots, Q_{10}
$$

of $\mathbb{R}^{4}$ of norm not exceeding $M$, if $\left\|T_{i}-Q_{i}\right\|<\delta$ for all $1 \leq i \leq 10$, then we have for the distance of products

$$
\left\|T_{1} \ldots T_{10}-Q_{1} \ldots Q_{10}\right\|<\epsilon
$$

By Approximation Lemma 5, there is a positive integer $l$ such that for all integers $k \geq l$ the set $\mathcal{L} \mathbb{R} C_{k}$ is $\delta$-dense within each of the three elementary space rotation groups around the standard space unit vectors, and also within the group of basic boosts of norm not exceeding $M$.

Let $T$ be any orthochronous proper Lorentz transformation, $T=R S B_{\alpha} S^{-1}$ where $R, S$ are space rotations, and suppose that the norm $\alpha+\alpha^{-1}$ of $T$ is at most $M$. We can factorize $R$ as a composition $R_{1} R_{2} R_{3}$ of three elementary space rotations, and factorize also as $S$ as the composition $R_{4} R_{5} R_{6}$ of three elementary space rotations. For convenience write $R_{7}=R_{6}^{-1}, R_{8}=$ $R_{5}^{-1}, R_{9}=R_{4}^{-1}$, so that

$$
T=R_{1} \ldots R_{6} B_{\alpha} R_{7} R_{8} R_{9}
$$

By $\delta$-density, there are elementary space rotations $R_{1}^{\prime}, \ldots, R_{9}^{\prime}$ with coefficients in $C_{k}$ and a basic boost $B^{\prime}$ also in $\mathcal{L}_{+} \mathbb{R} C_{k}$ and of norm at most $M$, such that for each $0 \leq i \leq 9$ the elementary rotations $R_{i}$ and $R_{i}^{\prime}$ have a common fixed standard space unit vector, and the distances

$$
\left\|R_{1}^{\prime}-R_{1}\right\|, \ldots,\left\|R_{9}^{\prime}-R_{9}\right\|,\left\|B^{\prime}-B_{\alpha}\right\|
$$

are all less than $\delta$. Then the distance of $T$ from the composition $R_{1}^{\prime} \ldots R_{6}^{\prime} B_{\alpha} R_{7}^{\prime} R_{8}^{\prime} R_{9}^{\prime}$ is at most $\epsilon$.

Conclusion of proof of Theorem 1 Given $\epsilon>0$ and $M>0$, by Approximation Lemma 6 we can take a positive integer $k$ such that the set $\mathcal{L}_{+} \mathbb{R} C_{k}$ is $\epsilon$-dense within the set of orthochronous proper Lorentz transformations of norm at most $M$.

By the Symmetrized Chain Lemma, there is a prime $p$,

$$
p>32 k^{16}>k, \quad p \equiv 7 \bmod 8
$$


such that the canonical surjection $\mathbb{Z}_{(p)} \longrightarrow \mathbf{F}_{p}$ maps all of $C_{k}$ inside the group of non-zero squares of $\mathbf{F}_{p}$. Then the canonical surjection $\mathcal{L}_{+} \mathbb{R} \mathbb{Z}_{(p)} \longrightarrow \mathcal{L}_{+} \mathbf{F}_{p}$ maps all of $\mathcal{L}_{+}^{\uparrow} \mathbb{R} C_{k}$ inside $\mathcal{L}_{+}^{\uparrow} \mathbf{F}_{p}$. Let $A=\mathcal{L}_{+}^{\uparrow} \mathbb{R} C_{k}$.

Define the retraction $f: \mathcal{L}_{+}^{\uparrow} \mathbb{R} \longrightarrow A$ by associating to each orthochronous proper Lorentz transformation $T$ a member of $A$ at minimum distance from $T$. Then apply the Local Isomorphism Lemma of 2.3.

\section{Extended Lorentz groups and line reflections}

Given $\epsilon>0$ and $M>0$ the retraction of the proper orthochronous Lorentz group $\mathcal{L}_{+}^{\uparrow} \mathbb{R}$ provided by Theorem 1 can in fact be defined also on the other three connected components of the extended Lorentz group, with the retract $A$ being locally isomorphic to a subset $Y$ of the extended Lorentz group $\mathcal{L} \mathbf{F}_{p}$ of all bijective linear transformations of $\mathbf{F}_{p}^{4}$ preserving the Minkowski norm. The local isomorphism between $A$ and $Y$ can also be seen to preserve several other properties of transformations besides the property of being orthochronous.

As over $\mathbb{R}$, over any field $\mathbf{F}$ (where -1 is not a square) the proper Lorentz group is an index 2 subgroup of the extended group $\mathcal{L} \mathbf{F}$, and we denote by $\mathcal{L}_{-} \mathbf{F}$ the coset consisting of the transformations of determinant -1 , called improper transformations. Among these we have in particular the time reversal transformation $\tau$ given by

$$
\tau(t, x, y, z)=(-t, x, y, z)
$$

Similarly to orthochronous transformations in $\mathcal{L}_{+} \mathbf{F}$, a transformation in $\mathcal{L}_{-} \mathbf{F}$ is also called orthochronous if it maps $(1,0,0,0)$ to a vector $(t, x, y, z)$ where $t$ is a non-zero square in $\mathbf{F}$. The set of orthochronous improper Lorentz transformations is denoted by $\mathcal{L}_{-}^{\uparrow} \mathbf{F}$.

Among all improper Lorentz transformations, a fundamental role is played by line reflections in time-like or space-like lines, i.e. 1-dimensional subspaces of $\mathbf{F}^{4}$ consisting of the scalar multiples of a vector $\mathbf{a} \in \mathbf{F}^{4}$ with Minkowski norm $\mu(\mathbf{a})$ equal to 1 or -1 . In the classical case $\mathbf{F}=\mathbb{R}$ this role was recently emphasized by Urbantke in [U]. Over any field $\mathbf{F}$, reflection in the 
line of $\mathbf{a}=\left(a_{0}, a_{1}, a_{2}, a_{3}\right) \in \mathbf{F}^{4}$ (where $\left.\mu(\mathbf{a})= \pm 1\right)$ is the improper Lorentz transformation with matrix

$$
2 \mu(\mathbf{a})\left[\mathbf{a}^{\top} \tau(\mathbf{a})-\mathbf{I}\right]
$$

where in the square bracket the matrices $\mathbf{a}^{\top}, \tau(\mathbf{a})$ and $\mathbf{I}$ are $4 \times 1,1 \times 4$ and $4 \times 4$, respectively, and $\tau$ is time reversal.

Theorem 2 For every $\epsilon>0$ and $M>0$ the extended Lorentz group $\mathcal{L} \mathbb{R}$ has a retraction $f: \mathcal{L} \mathbb{R} \longrightarrow A, f^{2}=f$, onto a finite subset $A \subseteq \mathcal{L} \mathbb{R}$ satisfying

$$
\|T-f(T)\|<\epsilon
$$

for all Lorentz transformations $T \in \mathcal{L} \mathbb{R}$ of norm not exceeding $M$, and such that for some prime number $p \equiv 7 \bmod 8$ the retract $A$ is locally isomorphic to a subset of $\mathcal{L F}_{p}$ via a local isomorphism $\lambda$, where the retraction $f$ and the local isomorphism $\lambda$ satisfy the following conditions:

(i) $f$ maps each of the 4 connected components of $\mathcal{L} \mathbb{R}$ to its intersection with $A$,

(ii) $\lambda$ maps each of the intersection sets

$$
A \cap \mathcal{L}_{+}^{\uparrow} \mathbb{R}, \quad A \cap \mathcal{L}_{+}^{\downarrow} \mathbb{R}, \quad A \cap \mathcal{L}_{-}^{\uparrow} \mathbb{R}, \quad A \cap \mathcal{L}_{-}^{\downarrow} \mathbb{R}
$$

respectively into the corresponding cosets

$$
\mathcal{L}_{+}^{\uparrow} \mathbf{F}_{p}, \quad \mathcal{L}_{+}^{\downarrow} \mathbf{F}_{p}, \quad \mathcal{L}_{-}^{\uparrow} \mathbf{F}_{p}, \quad \mathcal{L}_{-}^{\downarrow} \mathbf{F}_{p}
$$

(iii) $\lambda$ maps space rotations to space rotations, boosts to boosts, line reflections to line reflections.

The extended retraction and local isomorphism of Theorem 2 are constructed similarly to those of Theorem 1. Generating the extended finite Lorentz group $\mathcal{L} \mathbf{F}_{p}$ by reflections now leads to a representation of the extended finite Lorentz group $\mathcal{L} \mathbf{F}_{p}$ as a quotient of the group of all Minkowski norm preserving automorphisms of the module $\mathbb{Z}_{(p)}^{4}$.

\section{Remarks on finiteness and approximation}


It has been noted (see Beltrametti and Blasi [BB2]) that, as opposed to $\mathcal{L}_{+}^{\uparrow} \mathbb{R}$ and $\mathcal{L}^{\uparrow} \mathbb{R}=\mathcal{L}_{+}^{\uparrow} \mathbb{R} \cup \mathcal{L}_{-}^{\downarrow} \mathbb{R}$, the set of orthochronous Lorentz transformations over a finite field does not constitute a group. For example, take any prime number $p \equiv 7 \bmod 8$ and such that 3 is a quadratic residue modulo $p$. The first positive integer $q$ such that $q+1$ is not a quadratic residue modulo $p$ is even, and $q / 2$ is then a quadratic residue. Let $\alpha$ and $\gamma$ be non-zero squares in $\mathbf{F}_{p}$ such that the elements of $\mathbf{F}_{p}$ corresponding to the integers 2 and $q / 2$ are $\alpha^{2}$ and $\gamma^{2}$ respectively. Then the basic boosts $B_{\alpha}$ and $B_{\gamma}$ are orthochronous but $B_{\alpha} B_{\gamma}=B_{\alpha \gamma}$ is not. However, for all orthochronous transformations $T_{1}, T_{2}$ in the set $Y \subseteq \mathcal{L}_{+} \mathbf{F}_{p}$ of Theorem 1, the construction of the local isomorphism between $A \subseteq \mathcal{L}_{+}^{\uparrow} \mathbb{R}$ and $Y$ implies that $T_{1} T_{2}$ is also orthochronous. Thus the phenomenon of an antichronous product of two orthochronous parallel boosts arises only when the factors are not confined to the set $Y$ which in the Lorentz group over $\mathbf{F}_{p}$ represents, in the sense of local isomorphism, the retract $A \subseteq \mathcal{L}_{+}^{\uparrow} \mathbb{R}$.

In contrast to parallel boost groups in $\mathcal{L} \mathbb{R}$, in $\mathcal{L} \mathbf{F}_{p}$ the boost group

$$
\left\{R B_{\alpha} R^{-1}: \alpha \neq 0, \alpha \text { square in } \mathbf{F}_{p}\right\}
$$

is cyclic for any space rotation $R$, and it is isomorphic to the multiplicative subgroup of non-zero squares in $\mathbf{F}_{p}$ via the map $\alpha \mapsto R B_{\alpha} R^{-1}$. The finite model then involves in each space direction the existence of a boost from which all parallel boosts in that direction are obtainable by repeated application.

As in the real number based model, over a finite field $\mathbf{F}_{p}$ as well the velocity $v_{\alpha}$ associated with the basic boost $B_{\alpha}$ is $\left(\alpha-\alpha^{-1}\right) /\left(\alpha+\alpha^{-1}\right)$ and for the velocities of boosts $B_{\alpha}$ and $B_{\gamma}$ and of their composition $B_{\alpha \gamma}$ we have

$$
v_{\alpha \gamma}=\frac{v_{\alpha}+v_{\gamma}}{1+v_{\alpha} v_{\gamma}}
$$

In finite field models as well as over $\mathbb{R}$, velocity never equals 1 . For $p \equiv$ $7 \bmod 8$, if $\alpha^{2}+1$ is a square in $\mathbf{F}_{p}$ (and this is always the case if $B_{\alpha}$ is in the set $Y \subseteq \mathcal{L}_{+}^{\uparrow} \mathbf{F}_{p}$ locally isomorphic to the retract $A \subseteq \mathcal{L}_{+}^{\uparrow} \mathbb{R}$ as in Theorem 1 ), then $1-v_{\alpha}$ is a square in $\mathbf{F}_{p}$ i.e. in the non-transitive order $<_{p}$ on $\mathbf{F}_{p}$ considered by Kustaanheimo in [K1, K2] and given by

$$
x<_{p} y \Leftrightarrow y-x \text { is a non-zero square in } \mathbf{F}_{p}
$$


we have

$$
-1<_{p} v_{\alpha}<_{p} 1
$$

Velocities $v_{\alpha}$ not constrained by these inequalities in $\mathbf{F}_{p}$ may only arise from boosts in $\mathcal{L} \mathbf{F}_{p}$ that do not correspond, via the local isomorphism of Theorem 1 , to boosts in the retract $A \subseteq \mathcal{L}_{+}^{\uparrow} \mathbb{R}$ which approximate all boosts of the real model of norm not exceeding $M$ i.e. real boosts of speed not exceeding $\left(1-4 / M^{2}\right)^{1 / 2}$. As the bound $M$ can be as large as needed, this means that superluminal velocity $\left(1<_{p} v_{\alpha}\right.$ in $\left.\mathbf{F}_{p}\right)$ cannot arise from boosts in $\mathcal{L} \mathbf{F}_{p}$ that correspond to boosts in $\mathcal{L} \mathbb{R}$ with speed effectively distinguishable from the speed of light.

\section{References}

[A1] Y. Ahmavaara, Relativistic quantum theory as a group problem, II. World geometry and the elementary particles, Annales Ac. Sc. Fennicae A VI Physica 95 (1962) 51pp

[A2] Y. Ahmavaara, The structure of space and the formalism of relativistic quantum theory, I-IV, J. Math. Phys. 4 (1965) 87-93, 6 (1965) 220-227, 7 (1966) 197-201, 7 (1966) 201-204

[BB1] E.G. Beltrametti, A.A. Blasi, Dirac spinors, covariant currents and the Lorentz group over a finite field, Nuovo Cimento LVA/2 (1968) 301-317

[BB2] E.G. Beltrametti, A. Blasi, Rotation and Lorentz groups in a finite geometry, J. Math. Phys. 9 (1968) 1027-1035

[BGZG] A.A. Blasi, F. Gallone, A. Zecca, V. Gorini, A causality group in finite space-time, Nuovo Cimento 10A/1 (1972) 19-36

[C] H.R. Coish, Elementary particles in a finite world geometry, Phys. Rev. 114 - 1 (1959) 383-388

[D] L.E. Dickson, Determination of the structure of all linear homogeneous groups in a Galois field which are defined by a quadratic invariant, American J. Math. 21 (1899) 193-256

[Jo] H. Joos, Group-theoretical models of local-field theories, J. Math. Phys. 5 (1964) 155-164 
[Jä] G. Järnefelt, Reflections on a finite approximation to euclidean geometry. Physical and astronomical prospects. Annales Ac. Sc. Fennicae A I. Math.-Phys. 96 (1951) 1-43

[JK] G. Järnefelt, P. Kustaanheimo, An observation on finite geometries, in Proc. Skandinaviske Matematikerkongress i Trondheim 1949, 166-182

[K1] P. Kustaanheimo, A note on a finite approximation of the euclidean plane geometry, Comment. Phys.-Math. Soc. Sc. Fenn. XV. 19 (1950) 1-11

[K2] P. Kustaanheimo, On the fundamental prime of a finite world, Annales Ac. Sc. Fennicae A I. Math.-Phys. 129 (1952) 1-7

$[\mathrm{M}] \mathrm{V}$. Moretti, The interplay of the polar decomposition theorem and the Lorentz group, in Lecture Notes of Seminario Interdisciplinare di Matematica 5-153 (2006) 18 pages, also arXiv:math-ph/0211047v1 at www.arxiv.org

$[N]$ Y. Nambu, Field theory of Galois fields, in Field Theory and Quantum Statistics, eds. J.A. Batalin et.al., Institute of Physics Publishing 1987, pp. $625-636$

[S] I.S. Shapiro, Weak interactions in the theory of elementary particles with finite space, Nuclear Phys. 21 (1960) 474-491

[U] H.K. Urbantke, Lorentz transformations from reflections: some applications, Found. Phys. Lett. 16 (2003) 111-117

[Y] Q.A.M. Yahia, Leptonic decays in finite space, Nuovo Cimento XXIX/2 (1963) 441-450 